\title{
PNEUMOPERICÁRDIO APÓS TRAUMATISMO PENETRANTE: RELATO DE TRÊS CASOS
}

\author{
PNEUMOPERICARDIUM AFTER PENETRATING TRAUMA: \\ REPORT OF THREE CASES
}

\author{
Fernando Antonio Campelo Spencer Netto, TCBC-PE ${ }^{1}$ \\ Jurandir Cavalcanti de Araújo Melo ${ }^{2}$ \\ FábioAntonioAmando Granja ${ }^{3}$
}

\section{INTRODUÇÃO}

Pneumopericárdio, acúmulo de ar ou gás no saco pericárdico, entidade pouco freqüente, pode ocorrer em decorrência de traumatismos, patologia em órgão adjacente que contenha ar ou gás, infecção, iatrogenias ${ }^{1,2}$.

Cummings, em revisão de literatura estudou 252 pacientes com pneumopericárdio, observando entre estes, 17 casos $(6,75 \%)$ decorrentes de traumatismo penetrante torácico ${ }^{2}$.

No Hospital da Restauração (HR) - Recife - Brasil, no primeiro semestre de 1998 foram observados três casos de pneumopericárdio por lesão penetrante de tórax, que são discutidos a seguir em seus aspectos fisiopatológicos, diagnósticos e terapêuticos.

\section{RELATO DOS CASOS}

\section{Caso 1}

MJS, sexo feminino, 17 anos, atendida na Emergência de Adultos do HR em 01/02/98, vítima de agressão por arma de fogo com orifício de penetração em hemitórax esquerdo, no terceiro espaço intercostal, em linha hemiclavicular, uma hora antes. Apresentava-se eupnéica, hidratada e normocorada, com pulsos firmes. A ausculta respiratória mostrou um murmúrio vesicular presente bilateralmente. A ausculta cardíaca não revelou alterações. A radiografia de tórax inicialmente não demonstrou alterações, estando o projétil em projeção de seio cardiofrênico esquerdo. Radiografia repetida com $12 \mathrm{~h}$, apresentava os mesmos achados, tendo a paciente recebido alta em seguida. No terceiro dia pós-trauma, a paciente retorna com dor à inspiração, sendo identificado pneumopericárdio à radiografia de tórax (Figura 1). Ecocardiograma demonstrou pneumopericárdio leve, com demais parâmetros normais. Optou-se pela conduta conservadora e alta, estando a paciente assintomática no seguimento de sete dias.

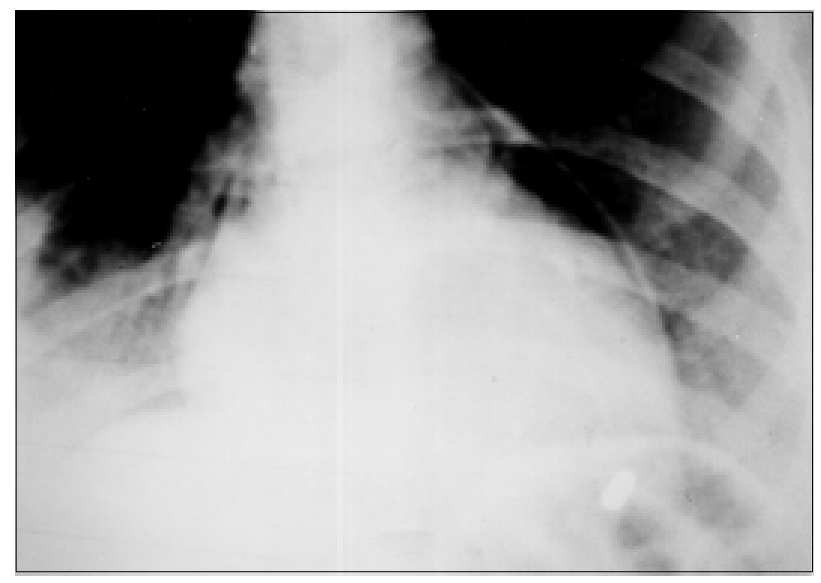

Figura 1 - Pneumopericárdio produzido por lesão penetrante por projétil de arma de fogo.

\section{Caso 2}

EJB, sexo masculino, 18 anos, atendido na Emergência de Adultos do HR em 10/04/98, vítima de agressão por arma branca (duas lesões) sendo uma delas em hemitórax direito no sétimo espaço intercostal, medialmente a linha hemiclavicular e a segunda em hipocôndrio esquerdo. Apresentava-se alcoolizado, com vias aéreas pérvias, mur-

1. Cirurgião Geral do Hospital da Restauração. Instrutor do ATLS/PE. Mestre em Cirurgia - UFPE.

2. Acadêmico Medicina - UFPE.

3. Residente em Cirurgia Geral do Hospital da Restauração - SUS/PE.

Recebido em 27/09/2000

Aceito para publicação em 31/07/2001

Trabalho desenvolvido na Emergência de Adultos do Hospital da Restauração — Recife. 
múrio vesicular diminuído à direita, hipocorado (+/+4), pulsos firmes. Realizada a drenagem torácica subaquática à direita, com saída imediata de $300 \mathrm{ml}$ de conteúdo sanguinolento e escape aéreo. A exploração da ferida em hipocôndrio esquerdo não revelou penetração em abdome. O exame radiológico de controle demonstrou expansão pulmonar e pneumopericárdio (Figura 2). O paciente permaneceu estável após a drenagem evoluindo na enfermaria com escape aéreo persistente, tratado com aspiração contínua. Ao oitavo dia de internação hospitalar foi retirado dreno pleural, com radiografia de controle normal e o paciente obteve alta hospitalar.

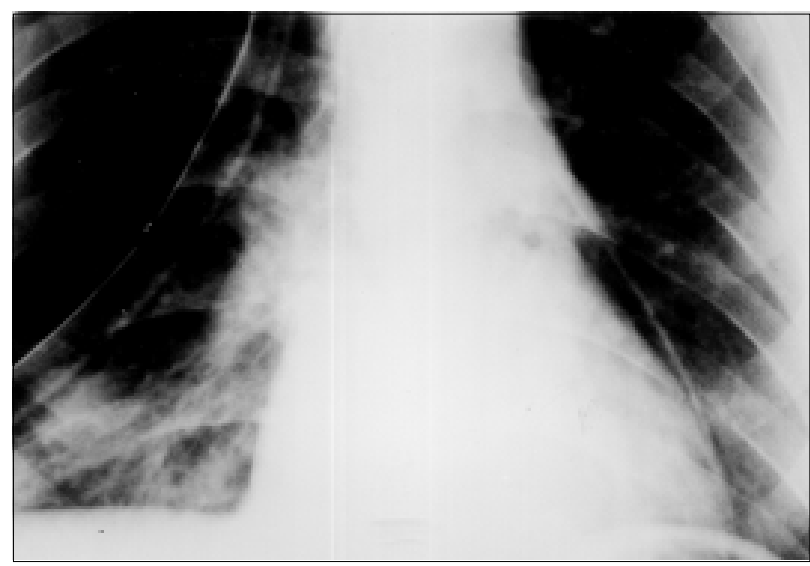

Figura 2 - Radiografia demonstrando drenagem torácica em hemitórax direito e pneumopericárdio.

\section{Caso 3}

EJS, sexo masculino, 27 anos, foi atendido na Emergência de Adultos do HR em 18/04/98 após agressão por arma branca em região toracoabdominal direita, próxima ao apêndice xifóide e em flanco direito. $\mathrm{O}$ murmúrio vesicular estava diminuído no hemitórax direito, e a radiografia de tórax revelou derrame pleural à direita e pneumopericárdio (Figura 3). Executada drenagem torácica em selo d'água, com saída de aproximadamente $300 \mathrm{ml}$ de sangue e ar. A ausculta cardíaca permaneceu normal durante todo período. $\mathrm{O}$ ferimento no flanco direito não apresentava penetração para cavidade peritoneal. Paciente manteve-se estável, evoluindo sem intercorrências. No quinto dia de internamento hospitalar uma nova radiografia mostrava um hemotórax residual discreto. No sétimo dia de internação foi retirado o dreno, com radiografia de tórax normal, o paciente recebeu alta hospitalar.

\section{DISCUSSÃO}

O pneumopericárdio traumático pode ser causado por feridas penetrantes ou traumatismo contuso. Quando excluídos os barotraumas causados por ventilação mecânica, torna-se infreqüente o trauma fechado como causa de pneumopericárdio ${ }^{1}$. Estes podem decorrer de lesões nas vias

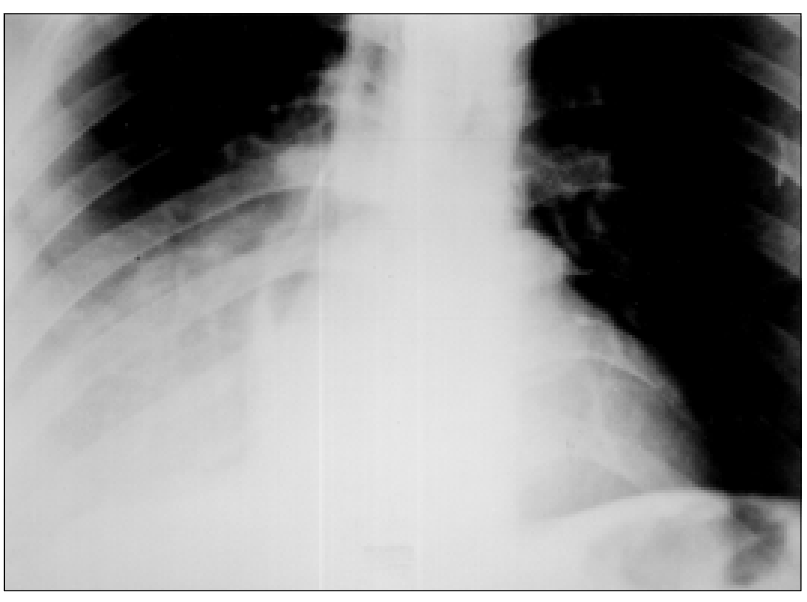

Figura 3 - Radiografia de tórax demonstrando hemotórax 'a direita e pneumopericárdio.

aéreas ou parênquima pulmonar. Nestes casos, usualmente observa-se concomitante pneumotórax que se comunica através de lesão traumática com o pericárdio ou, quando na ausência de lesão pericárdica, por dissecção pelo ar do espaços perivasculares dos vasos pulmonares ${ }^{1}$.

A formação do pneumopericárdio no trauma penetrante do tórax, ocorre por lesão direta e entrada do ar atmosférico através da ferida cutânea. Alternativamente, pode ocorrer lesão visceral associada, usualmente o pulmão, estruturas que se comunicam com a cavidade pericárdica pela lesão do saco pericárdico. Geralmente é acompanhado de derrame pleural como nos casos II e III, aqui relatados. $\mathrm{Na}$ presença de aderências pulmonares ou de lesões de vias aéreas mediastinais, o derrame pleural pode não ser observado ${ }^{2}$.

Na primeira e ampla revisão sobre o assunto, Cummings et al. estudaram 17 casos decorrentes de traumatismo torácico penetrante entre 252 pacientes $^{2}$. Desde então, foram publicadas inclusive séries de pacientes com pneumopericárdio produzidos por arma branca ${ }^{3,4}$. São raros os relatos de pneumopericárdio produzido por arma de fogo $^{2,3}$.

Demetriades detem a maior casuística de pacientes portadores de pneumopericárdio causados por traumatismo penetrante, ao todo 20 casos produzidos por arma branca ${ }^{4}$. O mesmo autor questiona a raridade do pneumopericárdio após traumatismo penetrante. Em revisão de casuística pessoal de 70 casos de trauma penetrante de coração por arma branca, com exame radiológico em 17 pacientes, encontrou pneumopericárdio em cinco o que corresponde a $15 \%$ dos investigados ${ }^{3,4}$. Saliente-se que na condição de emergência, apenas aqueles pacientes com estabilidade hemodinâmica são submetidos a exame radiológico. Entre estes, o diagnóstico de pneumopericárdio pode passar despercebido por desenvolver-se em momento posterior ao exame inicial, de forma assintomática ou mesmo por não ser percebido na interpretação inicial do exame radiológico.

A presença do pneumopericárdio após traumatismo penetrante indica lesão pericárdica e alta possibilidade de 
lesão miocárdica subjacente, devido a sua contiguidade anatômica, sendo a lesão do pericárdio considerada como lesão cardíaca por alguns autores 5 . Quando presente, independente de lesão miocárdica concomitante, a lesão do pericárdio permite o acúmulo anormal de fluidos na cavidade pericárdica. A presença do pneumopericárdio isolado após trauma penetrante raramente traz morbidade, sendo incomum a formação de mecanismo valvular unidirecional no nível de pericárdio determinando regime de hipertensão e quadro clínico de tamponamento cardíaco ${ }^{3}$. Para ocorrerem efeitos hemodinâmicos é necessário que o aumento de pressão intrapericárdica, determinado pelo ar, atinja níveis de $145 \mathrm{mmH}_{2} \mathrm{O}$, o que corresponde a cerca de $60 \mathrm{ml}$ de gás injetado agudamente. $\mathrm{O}$ tamponamento desenvolve-se com pressões acima de $265 \mathrm{mmH}_{2} \mathrm{O}^{2}$. O acúmulo lento de fluido permite que a cavidade pericárdica abrigue grandes volumes sem aumento importante de pressão pericárdica ${ }^{2}$.

Ressalte-se que a utilização de ventilação mecânica pode exacerbar a fístula aérea, levando a regime de hipertensão no pneumopericárdio ${ }^{1}$.

O quadro clínico de um paciente com pneumopericárdio produzido por traumatismo penetrante é variável, sendo usualmente resultado das lesões subjacentes, como nos casos II e III, onde o derrame pleural foi o responsável pela sintomatologia. O pneumopericárdio só provocará sintomatologia, por si próprio, quando determinar aumento significativo de pressão intrapericárdica, com quadro de tamponamento. Nessas circunstâncias, ao exame físico pode-se observar timpanismo na área cardíaca, e o sinal auscultatório clássico em "ruído de moinho" descrito por Bricheteau como "semelhante às pás de um moinho batendo na água" ${ }^{2}$. Não foram anotadas alterações específicas da ausculta nos casos aqui relatados.

O eletrocardiograma pode ser normal, demonstrar alterações sugestivas de pericardite ou alterações inespecíficas de segmento ST e ondas T. Com tamponamento, há baixa voltagem detectada em todas as derivações ${ }^{2}$. O ecocardiograma é o exame de escolha para a detecção de possível lesão cardíaca associada. Não é, entretanto, usualmente disponível na maioria dos serviços de emergência.

A avaliação radiológica, através da radiografia simples do tórax, é o meio diagnóstico mais utilizado, pela simplicidade de execução e pela especificidade, sendo o método usado na totalidade dos pacientes estudados. O pneumopericárdio é reconhecido radiologicamente devido à transparência entre o coração e ao pericárdio salientado pela presença do ar, situando-se a porção superior desta imagem no nível de vasos da base, inferior ao joelho aórtico e delimitando a borda cardíaca esquerda, havendo possibilidade de mudança da coluna aérea com o decúbito ${ }^{1,4}$. $\mathrm{Na}$ projeção lateral observa-se transparência anterior ao coração e base dos grandes vasos. O diagnóstico diferencial é feito basicamente com o pneumomediastino. Neste, a coluna aérea é observada acima do joelho aórtico, delimitando vasos da base e traquéia com freqüência, determinando por vezes enfisema subcutâneo, e sem mudança da coluna aérea com o decúbito ${ }^{1,4}$.

A simples presença do pneumopericárdio é considerada critério de indicação cirúrgica, para alguns autores pela possibilidade de lesão cardíaca associada. Esta conduta, entretanto, vem sendo atualmente alvo de críticas, com alguns trabalhos demonstrando tratamento conservador bem-sucedido, resultado compatível com os observados nos pacientes aqui relatados ${ }^{4,5}$.

A conduta terapêutica a ser adotada deverá ser orientada pelas lesões associadas, uma vez que o pneumopericárdio simples usualmente não determina sintomatologia. Considera-se entretanto que a simples detecção do pneumopericárdio obriga a vigilância contínua em serviço de emergência, pela possibilidade de desenvolvimento tardio de mecanismo de hipertensão pericárdica sendo anotados casos com instalação em até $48 \mathrm{~h}^{1}$. No caso I da presente série, a paciente recebeu alta $24 \mathrm{~h}$ após a agressão, com exame radiológico normal, retornando no terceiro dia após o trauma com queixa de dor torácica, provavelmente relacionada ao pneumopericárdio. É possível que esteja envolvida uma lesão de segundo tempo, provocada pela necrose de tecido lesado pela passagem do projétil.

Quando em regime de hipertensão no espaço pericárdico, poderá ser utilizada a pericardiocentese como procedimento inicial até a instituição de drenagem pericárdica ou toracotomia. A decisão quanto ao procedimento definitivo estará na dependência da urgência do caso, possibilidade do controle da fístula aérea e lesões associadas com o procedimento escolhido e da experiência do Serviço $^{3}$. No presente estudo os três pacientes foram conduzidos sem necessidade de medida específica para o pneumopericárdio, sendo realizado apenas o tratamento das lesões associadas.

\begin{abstract}
Pneumopericardium after penetrating wound represents a high suspicion for cardiac wound. Some authors recommend thoracotomy to discharge a cardiac lesion. We present three cases of post-traumatic pneumopericardium one following a gunshot wound and two following a stab wound and discuss about diagnosis and treatment. None showed clinical signs of cardiac tamponade. Diagnosis was made by chest $x$-ray. Pneumopericardium was identified at the initial evaluation in two patients, who had concomitant hemothorax and underwent chest drainage. The patient with penetrating thoracic wound by gunshot pneumopericardium developed $24 \mathrm{~h}$ after trauma. Treatment was directed to the associated lesions without specific measurements for pneumopericardium. This aproach was safe in these patients.
\end{abstract}

Key Words: Penetrating thoracic trauma; Pneumopericardium. 


\section{REFERÊNCIAS}

1. Capizzi PJ, Martin M, Bannon MP. Tension pneumopericardium following blunt injury. J Trauma 1995; 39:775-80.

2. Cummings RG, Wesly RLR, Adams DH et al. Pneumopericardium resulting in cardiac tamponade. Ann Thorac Surg 1984; 37:511-18.

3. Demetriades D, Levy R, Hatzitheofilou C et al. Tension pneumopericardium following penetrating trauma: case report. J Trauma 1990; 30:238-9.

4. Demetriades D, Charalambides D, Pantanowitz D et al. Pneumopericardium following penetrating chest injuries. Arch. Surg. 1990;125:1187-9.
5. Michelow BJ, Bremner CG. Penetrating cardiac injuries: seletive conservatism - favorable or foolish? J Trauma 1987; 27:398-401.

Endereço para correspondência:

Rua Fernando Simões Barbosa, 50/102 - Boa Viagem 51020-390 - Recife-PE.

E-mail: kummerspencer@yahoo.com.br 